\title{
Virology, DNA Viruses, Papillomavirus
}

National Cancer Institute

\section{Source}

National Cancer Institute. Virology, DNA Viruses, Papillomavirus. NCI Thesaurus. Code C19116.

The study of papilloma viruses and the diseases that they cause. 